\title{
What do uncertainty-averse decision-makers believe? ${ }^{\star}$
}

\author{
Matthew J. Ryan \\ School of Economics, The Australian National University, Canberra, ACT 0200, AUSTRALIA \\ (e-mail: matthew.ryang@anu.edu.au)
}

Received: December 14, 1999; revised version: February 26, 2001

Summary. This paper introduces the concept of firm belief, which is proposed as a new epistemic model for a wide class of preferences. In particular, firm beliefs are shown to have the following desirable properties: (i) they are derived from preferences according to a plausible rule of epistemic inference; (ii) they satisfy standard logical properties; and (iii) tractable representations of firm belief are available for all (suitably continuous) biseparable preferences [13, 14], including the Choquet expected utility [30] and maxmin expected utility [16] classes. We also use firm belief to construct a generalization of Nash equilibrium for (twoplayer) normal form games.

Keywords and Phrases: Epistemic foundations of decisions, Biseparable preferences, Normal form games.

JEL Classification Numbers: C72, D81.

\section{Introduction}

In his derivation of subjective expected utility (SEU), Leonard Savage employed a behavioral conception of beliefs. Beliefs - and in particular, probabilistic judgements about events - are identified with a particular impetus for action.

\footnotetext{
* Versions of this paper have circulated under the title "Firm Belief". In revising the paper, I have benefitted from the perceptive comments of Simon Grant, Massimo Marinacci, Stephen Morris, Sujoy Mukerji, and seminar audiences at the Australian National University, RUD 1999 (Université Paris I), Tilburg University and the University of Auckland. Particular thanks are owed to Rupa Athreya, Edi Karni and an anonymous referee for their careful reading of earlier drafts, and their many beneficial suggestions. Any remaining errors or weaknesses in the paper are, of course, entirely my own responsibility. I am also happy to acknowledge the financial assistance of the ARC through grant number A000000055.
} 
"If the state of mind in question is not capable of manifesting itself in some sort of extraverbal behavior, it is extraneous to our main interest. If, on the other hand, it does manifest itself through more material behavior, that should, at least in principle, imply the possibility of testing whether a person holds an event to be more probable than another, by some behavior expressing, and giving meaning to, his judgement." ([29, pp. 2728 (emphasis added)])

Savage succeeded in constructing a fully probabilistic model of beliefs, determined entirely from choice behavior. His construction requires only that preferences satisfy a small number of seemingly innocuous "consistency" restrictions.

Nevertheless, empirical challenges to the descriptive realism of Savage's preference restrictions quickly accumulated. Such evidence has inspired many generalizations of SEU which relax these restrictions. Savage's probabilistic beliefs have typically been lost in the process.

What are the epistemic motivations behind non-SEU preferences? Morris ([22]) addresses this question in some detail. He proposes three alternative behavioral notions of belief - Savage belief, strong belief and weak belief - and derives representations of these beliefs in the context of several well-known generalizations of SEU. His definitions are behavioral in the sense that beliefs are constructed directly from preferences, exactly in the spirit of Savage.

However, none of these epistemic models is entirely adequate for the analysis of two well-known preference classes: Choquet expected utility (CEU) preferences (axiomatized in various forms by Schmeidler ([30]), Gilboa [15], Sarin and Wakker [28], and others), and the maxmin expected utility (MEU) class of Gilboa and Schmeidler ([16]). These preference classes encompass various nonneutral attitudes to uncertainty, as exhibited, for example, in the experimental work of Ellsberg [10]. For this reason, there has been considerable recent interest in applications of the MEU and CEU models, and especially in game-theoretic applications.

Unfortunately, in the case of MEU and CEU, weak beliefs need not be logically coherent - belief in logical contradiction is possible; while strong and Savage belief are too conservative, particularly if one admits behavioral phenomena such as "uncertainty aversion". Therefore, the present menu of preference-based epistemic models is not very appetizing to those interested in applications of MEU or CEU.

The present paper offers a fourth alternative, called firm belief, which is designed to suit the palates of this group. Indeed, firm beliefs are suitable for all biseparable preferences $[13,14]$ - a wide class that includes CEU and MEU as special cases - provided they satisfy continuity on constant acts. Firm beliefs are logically coherent for all such preferences; but are less conservative than Savage or strong beliefs. Moreover, firm beliefs possess a tractable (and, we believe, interesting) characterization in terms of the canonical representation of a biseparable ordering. 
We also consider an application of firm belief to the study of normal form games. Firm equilibrium, a generalization of Nash equilibrium suitable for players with biseparable preferences, is defined in section 7. A "test" game devised by Kin Chung Lo in [20] is used to compare our solution concept to alternative generalizations of Nash equilibrium. ${ }^{1}$

\section{Preferences and beliefs}

Let $X$ be a set of outcomes, endowed with a topology with respect to which $X$ is connected, and let $\Omega$ be a finite set of states of the world. Actions (or simply "acts") will be mappings from states to outcomes; that is, elements of $X^{\Omega}$.

We adopt the following notational conventions throughout the paper. Given a partition $\left\{A_{1}, A_{2}, \ldots, A_{n}\right\}$ of $\Omega$, and an act $x^{i} \in X^{\Omega}$ for each $i \in\{1,2, \ldots, n\}$, the act $x_{A_{1}}^{1} x_{A_{2}}^{2} \ldots x_{A_{n-1}}^{n-1} x^{n} \in X^{\Omega}$ is defined as follows:

$$
x_{A_{1}}^{1} x_{A_{2}}^{2} \ldots x_{A_{n-1}}^{n-1} x^{n}(\omega)= \begin{cases}x^{1}(\omega) & \text { if } \omega \in A_{1} \\ x^{2}(\omega) & \text { if } \omega \in A_{2} \\ \vdots & \vdots \\ x^{n}(\omega) & \text { if } \omega \in A_{n} .\end{cases}
$$

If $x \in X$, then we also use $x$ to denote the constant act in $X^{\Omega}$ that assigns outcome $x$ to every state.

A preference ordering is a complete, transitive and non-trivial binary relation $\succeq$ on $X^{\Omega}$. That is, either $f \succeq g$ or $g \succeq f$ for any $f, g \in X^{\Omega} ; f \succeq h$ whenever $f \succeq g$ and $g \succeq h$; and $f \succ g$ for some $f, g \in X^{\Omega}$ (where $\succ$ denotes, as usual, the asymmetric part of $\succeq$ ).

Savage [29] evaluates the subjective probability of events (i.e. subsets of $\Omega$ ) by examining preferences. Event $E$ is defined to be no less (subjectively) probable than event $F$ if the decision-maker weakly prefers to "bet" on $E$ than on $F$; that is, $x_{E} y \succeq x_{F} y$ for some $x, y \in X$ such that $x \succ y$. Savage identifies conditions on preferences sufficient for this binary relation to constitute a qualitative probability. $^{2}$

For preferences which do not satisfy Savage's conditions, probabilistic beliefs may be untenable. Whether or not this is a serious problem depends on the application one has in mind. When studying normal form games, for example, what is important is to distinguish those strategies that are subjectively possible (from the point of veiw of some player) from those that are subjectively impossible. The former are typically subject to equilibrium restrictions: players must only entertain the possibility of rival strategies that are "reasonable". It is not crucial to game theory that the relative subjective likelihoods of possible strategies can be described using a probability.

\footnotetext{
${ }^{1}$ Further properties of firm equilibrium are studied in [27].

2 See [29, Theorem 1].
} 
In Savage's terms, state $\omega$ is subjectively possible precisely when the singleton event $\{\omega\}$ is non-null. In other words, when

$$
f_{\{\omega\}} h \succ g_{\{\omega\}} h \text { for some } f, g, h \in X^{\Omega} .
$$

Let $\mathscr{P}(\succeq)$ denote the set of states which satisfy (1). The event $E$ is Savage believed if $\mathscr{P}(\succeq) \subseteq E$. This is equivalent, in the SEU context, to $E$ being assigned subjective probability one.

Nevertheless, (1) is not the only plausible way to demarcate between subjectively possible and subjectively impossible states. Morris ([22]) offers two alternatives.

First, consider the set $\mathscr{P}^{S}(\succeq)$ of all states $\omega$ which satisfy the condition

$$
f_{\{\omega\}} h \succ g \text { for some } f, g, h \in X^{\Omega} \text { with } f\left(\omega^{\prime}\right) \succ g\left(\omega^{\prime}\right) \succ h\left(\omega^{\prime}\right) \forall \omega^{\prime} \in \Omega .
$$

Morris defines event $E$ to be strongly believed if $\mathscr{P}^{S}(\succeq) \subseteq E$. Strong belief has proved valuable in understanding perfect equilibrium behavior in normal form games (see [2],[3] and [22, Example 2]). However, for CEU and MEU preferences, strong and Savage belief are in fact equivalent ([22, Examples 3 and 4]).

Second, let $\mathscr{P}^{W}(\succeq)$ be the states which satisfy

$$
\begin{gathered}
\forall f, g \in X^{\Omega} \text { with } f\left(\omega^{\prime}\right) \succ g\left(\omega^{\prime}\right) \forall \omega^{\prime} \in \Omega, \\
f_{\{\omega\}} h \succ g \text { for some } h \in X^{\Omega} \text { such that } g\left(\omega^{\prime}\right) \succ h\left(\omega^{\prime}\right) \forall \omega^{\prime} \in \Omega .
\end{gathered}
$$

Event $E$ is weakly believed if $\mathscr{P}^{W}(\succeq) \subseteq E$. Weak belief provides a genuine alternative to Savage/strong belief in the MEU and CEU contexts, since $\mathscr{P}^{W}(\succeq)$ may be a proper subset of $\mathscr{P}(\succeq)$. However, it is possible that $\mathscr{P}^{W}(\succeq)$ may be empty when $\succeq$ is a member of the CEU or MEU class ([22, p.228]). Emptiness of $\mathscr{P}^{W}(\succeq)$ is problematic, as it implies weak belief in logical contradictions: both $E$ and $E^{c}$ are weakly believed, for any event $E .^{3}$ This is not to deny that very many CEU and MEU preferences induce perfectly acceptable weak beliefs, nor that weak belief has proved very useful in the analysis of games. ${ }^{4}$ Nevertheless, it is also clearly of value to obtain an epistemic model that generates logically coherent beliefs for all preferences in the MEU and CEU classes.

Savage/strong belief offers one such model. However, just as logical considerations dictate that the set $\mathscr{P}^{W}(\succeq)$ may be "too small" in some circumstances, several theorists have argued that the set $\mathscr{P}(\succeq)$ may be "too large", in the sense that the behavioral test for inclusion in this set is unduly weak. ${ }^{5}$ For example, if $x, y \in X, x \succ z$ and

${ }^{3}$ This conclusion, of course, assumes that the state space $\Omega$ is properly specified. Omitted states could also explain the apparent contradiction in beliefs. Section 6 contains further discussion of this point.

${ }^{4}$ See [25], [20] and [21].

${ }^{5}$ Game theorists studying non-SEU players have been particularly loathe to insist upon correct Savage beliefs about rivals' strategic behavior, arguing that "weaker" epistemic attitudes are often more plausible. See, for example, [8, p.312], [9, p.191], [18, p.8], [20, p.622 and Appendix] and [21, section 4.4]. 


$$
x \succ z_{\{\omega\}} x
$$

then $\{\omega\}$ is non-null. However, this is very circumstantial evidence on which to declare $\omega$ to be subjectively possible. Such behavior may simply reflect a psychological aversion to weakly dominated actions. For SEU preferences, additional, more compelling evidence can always be found to bolster the conclusion that $\omega$ is subjectively possible. In particular, when $\succeq$ are SEU preferences, $\mathscr{P}(\succeq)=\mathscr{P}^{S}(\succeq)=\mathscr{P}^{W}(\succeq)([22$, p.231]), so $\omega \in \mathscr{P}(\succeq)$ if and only if it also passes the more stringent test (3). The same need not be so of CEU or MEU preferences in general.

An intermediate model of belief - lying between weak and Savage/strong belief - is needed for CEU and MEU decision-makers. Firm belief, which is defined in the next section, provides one such model.

\section{Firm belief}

Consider the following definition, due to Lo [20]:

Definition 1 Given a preference ordering $\succeq$, and events $A, B \in 2^{\Omega}$, we say that $A$ is infinitely more likely than $B$, denoted $A \gg_{L} B$, if and only if

$$
x_{A} z \succ y_{B} z \quad \forall x, y, z \in X \text { with } x \succ z .
$$

This says that $A$ is infinitely more likely than $B$ if betting on $A$ is strictly preferable to betting on $B$, no matter how much better is the prize for winning the $B$ bet than that for winning the $A$ bet (with the consequences of losing the same in each case).

Lo developed this idea to study MEU preferences. It embodies the assumption of a "psychological desire to rule out completely the possibility of a misfortune" (ibid., p.625). If such a decision-maker believes that the true state lies in $A$, but not in $B$, then rich rewards offered in $B$ will not turn her head. This same decision-maker may nevertheless be wary of potential losses suffered in event $B$. In particular, $A \gg_{L} B$ need not imply that

$$
x_{A} z \succeq x_{A \cup B} z \quad \forall x, z \in X \text { with } x \succ z \text {. }
$$

If $B \neq \emptyset$, then the preference ranking in (5) violates strong monotonicity. ${ }^{6}$ By refraining from assigning undue epistemic significance to behavioral evidence such as violations of (5), Definition 1 may be used to construct a natural model of beliefs which is more permissive than Savage's.

Say that $\omega$ is subjectively possible if

$$
E \gg_{L} E \backslash\{\omega\} \text { for some } E \subseteq \Omega .
$$

\footnotetext{
${ }^{6}$ Preferences $\succeq$ are strongly monotone if $f \succ g$ whenever $f(\omega) \succeq g(\omega)$ for all $\omega \in \Omega$, and $f\left(\omega^{\prime}\right) \succ g\left(\omega^{\prime}\right)$ for some $\omega^{\prime} \in \Omega$.
} 
That is, $\omega$ is subjectively possible whenever there is an event $E$ such that removing $\omega$ from $E$ makes the event infinitely less likely. Let $\mathscr{P}^{F}(\succeq)$ denote the set of subjectively possible states associated with $\succeq$ under this definition. We shall say that an event $E$ is firmly believed if $\mathscr{P}^{F}(\succeq) \subseteq E$.

It is clear that $\{\omega\} \gg_{L} \emptyset$ implies (6) - take $E=\{\omega\}-$ but the converse is not true in general. Why do we favor the weaker test (6) over the stricter, but more straightforward, test $\{\omega\} \gg_{L} \emptyset$ ? The main reason is that the work of Lo [20] and Morris [22, Example 3] reveal that this latter route leads back to weak belief. Since some CEU and MEU preferences imply weak belief in logical contradiction; the test $\{\omega\} \gg_{L} \emptyset$ is too strict for our purposes.

Moreover, the lesson of Ellsberg [10] is that the behavioral manifestation of a state's subjective possibility may be strongest in contexts in which the state is critical to resolving some (subjective) uncertainty. Subjectively impossible states, on the other hand, will never be critical to resolving any such uncertainty. These observations provide additional motivation for (6). ${ }^{7}$

The remaining sections of the paper establish the credentials of firm belief as a logically coherent and tractable epistemic construct for all (suitably continuous) biseparable preferences [13, 14], including the CEU and MEU classes. We shall also verify that firm belief is strictly intermediate between weak and Savage/strong belief for the latter two classes. In fact, firm belief is, to our knowledge, the most conservative epistemic departure from Savage belief currently available for CEU or MEU preferences.

\section{Logical coherence}

Consider the class of preferences which admit the following lexicographic canonical representation. There exists a function $u: X \rightarrow \mathbb{R}$ and a mapping $\rho: \Omega \rightarrow \mathbb{R}^{n}$ for some $n \geq 1$ such that (i) $\rho(\Omega)=\mathbf{1}:=(1,1, \ldots, 1)$; (ii) $\rho(\emptyset)=\mathbf{0}:=(0,0, \ldots, 0)$; (iii) $A \subseteq B$ implies $\rho(A) \leq \rho(B)$; and (iv) for any $x, \hat{x}, y, \hat{y} \in X$ with $x \succeq y$ and $\hat{x} \succeq \hat{y}$, and any $A, B \in 2^{\Omega}$,

$$
x_{A} y \succeq \hat{x}_{B} \hat{y}
$$

if and only if

$$
\rho(A) u(x)+(\mathbf{1}-\rho(A)) u(y) \geq^{n} \rho(B) u(\hat{x})+(\mathbf{1}-\rho(B)) u(\hat{y})
$$

where $\geq^{n}$ is the usual lexicographic (partial) order on $\mathbb{R}^{n}$. Note that (7) implies $u$ represents $\succeq$ on constant acts. If $\succeq$ possesses a lexicographic canonical representation, we shall say that $\succeq$ is lexicographically biseparable.

The notion of a lexicographic canonical representation is a generalization of Ghirardato and Marinacci's [13, 14] canonical representation, which requires in addition that $n=1$ and the existence of a monotone extension of the representation to all acts. Similarly, Ghirardato and Marinacci introduced the term

\footnotetext{
${ }^{7}$ Yet another reason to favor (6) over $\{\omega\} \gg_{L} \emptyset$ is discussed in Section 6.
} 
biseparable to describe the preferences which have a canonical representation. Since the CEU and MEU orderings are biseparable ([14, Section 2.2]), they are also lexicographically biseparable. The latter class also includes Blume, Brandenburger and Dekel's [2] lexicographic expected utility preferences, and Klibanoff's [18] lexicographic extension of MEU.

The following result is proved in the Appendix. It verifies that firm belief is logically coherent for all lexicographically biseparable preferences that exhibit continuity on constant acts.

Theorem 1 If the preference ordering $\succeq$ possesses a lexicographic canonical representation with continuous $u$, then $\bar{P}^{F}(\succeq) \neq \emptyset$.

\section{Representation theorems}

\subsection{Biseparable preferences}

Given a state space $\Omega$, a capacity on $\Omega$ is a set function $v: 2^{\Omega} \rightarrow \mathbb{R}$, satsifying $v(\emptyset)=0, v(\Omega)=1$, and $v(E) \leq v(F)$ whenever $E \subseteq F$. For example, when $n=1$, the set function $\rho$ in a lexicographic canonical representation is a capacity. We can use properties of this capacity to characterize the firm beliefs underlying the associated preferences, provided the utility function $u$ in the representation is continuous. As a first step, we identify the connection between $\rho$ and Lo's "infinitely more likely than" relation.

Lemma 1 Let the preference ordering $\succeq$ possess a lexicographic canonical representation with $n=1$ and $u$ continuous. Then $A \gg_{L} B$ if and only if $\rho(A)>0$ and $\rho(B)=0$.

Proof. Suppose that $\rho(A)>0$ and $\rho(B)=0$. Then

$$
x_{A} z \succ y_{B} z
$$

for any $x, y, z \in X$ with $x \succ z$ and $y \succeq z$, since

$$
u(z)+\rho(A)[u(x)-u(z)]>u(z)=u(z)+\rho(B)[u(y)-u(z)] .
$$

Similarly, $x_{A} z \succ y_{B} z$ for any $x, y, z \in X$ with $x \succ z$ and $z \succ y$, since

$$
u(z)+\rho(A)[u(x)-u(z)]>u(z) \geq u(y)+\rho\left(B^{c}\right)[u(z)-u(y)] .
$$

Thus, $x_{A} z \succ y_{B} z$ for any $x, y, z \in X$ with $x \succ z$.

For the converse, suppose $A \gg_{L} B$. Fix $y, z \in X$ with $y \succ z$. Then

$$
\begin{gathered}
u(z)+\rho(A)[u(x)-u(z)]>u(z)+\rho(B)[u(y)-u(z)] \\
\Leftrightarrow \rho(B)<\frac{[u(x)-u(z)]}{[u(y)-u(z)]} \rho(A)
\end{gathered}
$$


for any $x \in X$ such that $x \succ z$. Since $\rho(B) \geq 0$, it follows from (8) that $\rho(A)>0$. Also, since $X$ is connected and $u$ continuous, $u(X)$ is an interval. Hence, $x$ can be chosen to make $u(x)-u(z)$ in (8) arbitrarily small, from which $\rho(B)=0$ follows.

The following definition is due to Dow and Werlang ([8, p.310]).

Definition 2 Given a capacity $v$ on $\Omega$, the set $A \subseteq \Omega$ is a support of $v$ if $A$ is $\subseteq$-minimal with respect to the property that $v\left(A^{c}\right)=0$. That is, $v\left(A^{c}\right)=0$, and $v\left(B^{c}\right)>0$ whenever $B$ is a proper subset of $A$.

If $v$ is a probability - that is, an additive capacity - then this notion of support coincides with the usual one. Furthermore, every capacity possesses a support, so Dow and Werlang's definition extends the notion of support from the class of probabilities to that of capacities. However, this extension fails to preserve uniqueness: some (non-additive) capacities have multiple supports ([8, p.311]).

Interestingly, if $\succeq$ is a biseparable preference ordering, then $\mathscr{P}^{F}(\succeq)$ is the union of the supports of the capacity $\rho$ (provided $u$ is continuous).

Theorem 2 Let the preference ordering $\succeq$ possess a lexicographic canonical representation with $n=1$ and $u$ continuous. Then $\mathscr{P}^{F}(\succeq)$ is the union of the supports of $\rho$.

Proof. Let $P$ denote the union of the supports of $\rho$. Applying Lemma 1, it suffices to show that $\omega \in P$ if and only if there exists some $E$ such that $\omega \in E, \rho(E)>0$ and $\rho(E \backslash\{\omega\})=0$.

For the "only if" part, let $A$ be a support of $\rho$ which contains $\omega$. Define $E=A^{c} \cup\{\omega\}$. Since $A$ is a support,

$$
\rho(E \backslash\{\omega\})=\rho\left(A^{c}\right)=0
$$

and $\rho(E)=\rho\left([A \backslash\{\omega\}]^{c}\right)>0$.

For the converse, we shall explicitly construct a support for $\rho$ which contains $\omega$. Begin with $A_{1}=E^{c} \cup\{\omega\}$. Note that $\rho\left(\left[A_{1}\right]^{c}\right)=\rho(E \backslash\{\omega\})=0$. If $\rho\left([F \cup\{\omega\}]^{c}\right)>0$ for every proper subset $F$ of $E^{c}$, then $A_{1}$ does the needful. Otherwise, if $\rho\left([F \cup\{\omega\}]^{c}\right)=0$ for some proper subset $F$ of $E^{c}$, let $A_{2}=F \cup\{\omega\}$. Continue in this fashion until reaching some set $A_{n}=B \cup\{\omega\}$, such that $B \subseteq E^{c}, \rho\left(\left[A_{n}\right]^{c}\right)=0$ and $\rho\left([H \cup\{\omega\}]^{c}\right)>0$ for every proper subset $H$ of $B$. Note that such an $A_{n}$ must eventually be reached, allowing for the possibility that $B=\emptyset$. The set $A_{n}$ will be a support for $\rho$ since $\rho\left(D^{c}\right) \geq \rho(E)>0$ for any $D \subseteq B$.

The following lemma offers a useful alternative characterization of firm beliefs:

Lemma 2 Let $v$ be a capacity on $\Omega$. The set $A \subseteq \Omega$ contains all the supports of $v$ if and only if $v(A \cap B)>0$ for any $B \subseteq \Omega$ with $v(B)>0$. 
Proof. For the "if" part, suppose that $v(A \cap B)>0$ whenever $v(B)>0$. In particular, $v(A)>0$ (take $B=\Omega$ ). Suppose further that $E$ is a support of $v$ that is not contained in $A$. It cannot be the case that $E$ is contained in $A^{c}$, since $v\left(E^{c}\right)=0$. Therefore, $E \cap A \neq \emptyset$. Let $F=E \cap A$. Since $F$ is a proper subset of $E, v\left(F^{c}\right)>0$. But $F^{c} \cap A=\emptyset$, and $v(\emptyset)=0$. Contradiction.

For the "only if" part, we shall again argue by contradiction. Therefore, let us assume that (i) $A$ contains all the supports of $v$; (ii) $v(B)>0$; and (iii) $v(A \cap B)=0$. Fact (ii) implies that there are no supports contained entirely in $A \cap B^{c}$. We shall obtain the desired contradiction by proving that (i) and (iii) imply the existence of a support in $A \cap B^{c}$. First, (iii) implies the existence of a support in $(A \cap B)^{c}$ as follows. If $(A \cap B)^{c}$ is not itself a support, then there exists some proper subset, call it $E_{1}$, with $v\left(E_{1}^{c}\right)=0$. If $E_{1}$ is not a support, we may similarly obtain a proper subset $E_{2}$ with $v\left(E_{2}^{c}\right)=0$. Since $v(\Omega)=1$, this process must eventually lead to a support. It will be contained in $(A \cap B)^{c}$. By (i), it will therefore also be contained in $A \cap B^{c}$.

Corollary 1 Let the preference ordering $\succeq$ possess a lexicographic canonical representation with $n=1$ and $u$ continuous. Then $\mathscr{P}^{F}(\succeq)$ is the intersection of all sets $A$ such that $\rho(A \cap B)>0$ whenever $\rho(B)>0$.

Finally, combining Theorem 2 and Corollary 1 we have:

Corollary 2 Let $\succeq$ be a biseparable preference ordering possessing a canonical representation with continuous $u$. Then

$$
\begin{aligned}
\mathscr{P}^{F}(\succeq) & =\bigcup\{A \subseteq \Omega \mid A \text { is a support of } \rho\} \\
& =\bigcap\{A \subseteq \Omega \mid \rho(A \cap B)>0 \text { whenever } \rho(B)>0\} .
\end{aligned}
$$

\subsection{Two important special cases: $C E U$ and $M E U$}

As has already been mentioned, CEU and MEU preferences are biseparable, so Corollary 2 covers these cases. The MEU orderings are those which may be represented as follows: for any $f, g \in X^{\Omega}$,

$$
f \succeq g \Leftrightarrow \min _{p \in C} p \cdot(u \circ f) \geq \min _{p \in C} p \cdot(u \circ g)
$$

where $u: X \rightarrow \mathbb{R}$ is a utility function, and $C$ is a closed, convex subset of $\Delta(\Omega)$, the set of all probabilities on $\Omega$. Such preferences therefore have a canonical representation with $\rho$ the "lower envelope" of $C$ :

$$
\rho(A)=\min _{p \in C} p(A) \quad \forall A \subseteq \Omega
$$

The MEU preferences were originally axiomatized by Gilboa and Schmeidler ([16]) within the framework of Anscombe and Aumann [1], in which $X$ is a mixture set of "roulette lotteries". More recently, Casadesus-Masanell, Klibanoff 
and Ozdenoren [5] have provided an axiomatization under which $X$ is required only to be connected and separable.

The MEU orderings all exhibit "uncertainty aversion". Within the framework of Anscombe-Aumann, this concept is typically understood in the following sense: ${ }^{8}$

Definition 3 (Uncertainty Aversion) For all acts $f, g \in X^{\Omega}$ and all $\alpha \in(0,1)$,

$$
f \sim g \Rightarrow \alpha f+(1-\alpha) g \succeq f .
$$

The definition reflects the potential psychological benefit of mixing two acts as a hedge against uncertainty. Casadesus-Masanell, Klibanoff and Ozdenoren [5] capture the same basic intuition within their own framework, using the concept of a standard sequence from measurement theory. Their paper may be consulted for a precise statement of the analogue to Definition 3, which is somewhat involved.

It should be noted, however, that alternative definitions of uncertainty (or ambiguity) aversion have also been proposed. Epstein [11] and Ghirardato and Marinacci [13] construct comparative definitions, in the spirit of Yaari's [32] approach to risk aversion. Although both comparative notions differ, each definition classifies the MEU preferences as uncertainty (or ambiguity) averse ([11, Theorem 3.3] and [13, Corollary 2]). MEU is therefore an important model of uncertainty-averse behavior.

Morris ([22, Example 3]) provides a representation of $\mathscr{P}(\succeq), \mathscr{P}^{S}(\succeq)$ and $\mathscr{P}^{W}(\succeq)$ for the MEU class. In particular, he demontrates that

$$
\mathscr{P}(\succeq)=\mathscr{P}^{S}(\succeq)=\bigcup_{p \in C} \operatorname{supp}(p)
$$

and

$$
\mathscr{P}^{W}(\succeq)=\bigcap_{p \in C} \operatorname{supp}(p)
$$

where $\operatorname{supp}(p)$ denotes the support of the probability $p$. Corollary 2 implies the following characterization of $\mathscr{P}^{F}(\succeq)$ in the MEU case.

Corollary 3 When $\succeq$ is an MEU ordering with u continuous,

$$
\begin{aligned}
\mathscr{P}^{F}(\succeq) & =\bigcup\left\{A \subseteq \Omega \mid A \text { is a support of } \rho \equiv \min _{p \in C} p\right\} \\
& =\bigcap\{A \subseteq \Omega \mid p(A \cap B)>0 \forall p \in C \text { whenever } p(B)>0 \forall p \in C\} .
\end{aligned}
$$

Combining this result with (11) and (12), it is simple to establish that

$$
\mathscr{P}^{W}(\succeq) \subseteq \mathscr{P}^{F}(\succeq) \subseteq \mathscr{P}^{S}(\succeq)=\mathscr{P}(\succeq)
$$

for all MEU preferences with continuous $u$.

The CEU model also embodies an array of uncertainty-averse behaviors, but unlike MEU, it allows other non-neutral attitudes to uncertainty to be captured

\footnotetext{
${ }^{8}$ See [16] and [30].
} 
as well. A preference ordering $\succeq$ is in the CEU class if there exists a utility function $u: X \rightarrow \mathbb{R}$ and a capacity $v$ on $\Omega$ such that, for any two acts $f$ and $g$,

$$
f \succeq g \quad \Leftrightarrow \quad \int_{\Omega}(u \circ f) d v \geq \int_{\Omega}(u \circ g) d v
$$

where integration is in the sense of Choquet [6].

Schmeidler [30] axiomatizes the CEU orderings within the AnscombeAumann framework. ${ }^{9}$ In this case, uncertainty aversion - in the sense of Definition 3 - is equaivalent to convexity of $v$; that is, $v(E \cup F)+v(E \cap F) \geq v(E)+v(F)$ for any $E, F \in 2^{\Omega}$ ([30]). However, convexity of the capacity is not equivalent to uncertainty/ambiguity aversion as defined by either Epstein [11] or Ghirardato and Marinacci [13]. ${ }^{10}$

Corollary 4 When $\succeq$ is a CEU ordering with u continuous,

$$
\begin{aligned}
\mathscr{P}^{F}(\succeq) & =\bigcup\{A \subseteq \Omega \mid A \text { is a support of } v\} \\
& =\bigcap\{A \subseteq \Omega \mid v(A \cap B)>0 \text { whenever } v(B)>0\} .
\end{aligned}
$$

Comparing Corollary 4 with [22, Example 4], one observes that

$$
\mathscr{P}^{W}(\succeq) \subseteq \mathscr{P}^{F}(\succeq) \subseteq \mathscr{P}^{S}(\succeq)=\mathscr{P}(\succeq)
$$

for all CEU preferences with continuous $u .^{11}$

In summary, for all MEU and CEU preferences with continuous utility on constant acts, firm belief is intermediate in strength between weak and Savage/strong belief. The examples in the next section show that firm belief lies strictly between these two poles.

\section{Examples}

Example 1 Consider MEU preferences with $C=\Delta(\Omega)$. In this case,

$$
f \succeq g \Leftrightarrow \min _{\omega \in \Omega}(u \circ f)(\omega) \geq \min _{\omega \in \Omega}(u \circ g)(\omega)
$$

and

$$
\mathscr{P}(\succeq)=\mathscr{P}^{S}(\succeq)=\mathscr{P}^{F}(\succeq)=\Omega,
$$

while $\mathscr{P}^{W}(\succeq)=\emptyset$. That is, weak belief is logically incoherent, while the other models of belief imply belief only in logical tautologies (i.e. propositions true in all states of the world).

\footnotetext{
${ }^{9}$ Various axiomatizations in other frameworks are also available: for example, [15] and [28].

${ }^{10}$ See also [31, Theorem 5.2].

11 Morris shows that

$$
\mathscr{P}^{W}(\succeq)=\left\{\omega \in \Omega \mid v(E \cup\{\omega\})>v(E) \quad \forall E \subseteq\{\omega\}^{c}\right\} .
$$
}

This implies in particular that $v(\{\omega\})>0$ for each $\omega \in \mathscr{P}^{W}(\succeq)$. Hence, if $\omega \in \mathscr{P}^{W}(\succeq)$, then $v(A)>0$ for every $A$ containing $\omega$, by the monotonicity of $v$. Therefore, $\mathscr{P}^{W}(\succeq)$ must be contained in every support of $v$. 
Example 2 Suppose that $A \subseteq \Omega$, with $1<|A|<|\Omega|$, and let the (convex) capacity $v$ be defined as follows: $v(\Omega)=1, v(E)=0.5$ if $A \subseteq E \neq \Omega$, and $v(E)=0$ otherwise. Then

$$
\int_{\Omega}(u \circ f) d v=\frac{1}{2} \min _{\omega \in \Omega}(u \circ f)(\omega)+\frac{1}{2} \min _{\omega \in A}(u \circ f)(\omega) .
$$

Consider the associated CEU/MEU preferences $\succeq$. One may easily show that

$$
\mathscr{P}(\succeq)=\mathscr{P}^{S}(\succeq)=\Omega \text { and } \mathscr{P}^{W}(\succeq)=\emptyset .
$$

That is, neither Savage/strong nor weak belief make any epistemic distinction between these preferences and those in the previous example. However, firm belief does so, as $\mathscr{P}^{F}(\succeq)=A$.

Example 2 shows that firm belief is not equivalent to any of the alternative epistemic models.

Observe that in each of the two previous examples, $\mathscr{P}^{W}(\succeq)=\emptyset$. Examples 1 and 2 are therefore situations in which weak beliefs are logically incoherent unless $\Omega$ is misspecified. Indeed, the capacities in Examples 1 and 2 are belief functions [7], and CEU with belief functions is sometimes interpreted as a model of decision-making based on an incomplete specification of the state space. ${ }^{12}$

However, the next example shows that firm beliefs may fail to coincide with weak beliefs, even when $\mathscr{P}^{W}(\succeq)$ is non-empty.

Example 3 Let $\Omega=\left\{\omega_{1}, \omega_{2}, \omega_{3}, \omega_{4}\right\}$. Let $C$ denote the convex hull of the set

$$
\begin{gathered}
\left\{\left(\frac{3}{4}, 0,0, \frac{1}{4}\right),\left(0,0, \frac{3}{4}, \frac{1}{4}\right),\left(\frac{1}{4}, \frac{1}{2}, 0, \frac{1}{4}\right),\right. \\
\left.\left(0, \frac{1}{2}, \frac{1}{4}, \frac{1}{4}\right),\left(\frac{1}{4}, 0,0, \frac{3}{4}\right),\left(0,0, \frac{1}{4}, \frac{3}{4}\right)\right\} \subseteq \Delta(\Omega) .
\end{gathered}
$$

If $\succeq$ are the preferences obtained from $C$ according to (9), then one may show that

$$
\begin{array}{r}
\mathscr{P}(\succeq)=\mathscr{P}^{S}(\succeq)=\Omega, \\
\mathscr{P}^{W}(\succeq)=\left\{\omega_{4}\right\}, \text { and } \mathscr{P}^{F}(\succeq)=\left\{\omega_{1}, \omega_{3}, \omega_{4}\right\} .
\end{array}
$$

Example 3 also illustrates another unfortunate property of weak belief. Observe that $\rho\left(\mathscr{P}^{W}(\succeq)\right)=\rho\left(\Omega \backslash \mathscr{P}^{W}(\succeq)\right)$ when $\rho \equiv \min _{p \in C} p$. In particular, the possessor of these preferences would be no more or less willing to "bet" on a weakly believed proposition than its negation. ${ }^{13}$ By contrast, for any biseparable preferences $\succeq$ with continuous $u$, firm belief shares with Savage belief the intuitive property that believed events are regarded as infinitely more likely than their negations. This follows from the facts that $\rho\left(\Omega \backslash \mathscr{P}^{F}(\succeq)\right)=0$ by Theorem 2 , and $\rho\left(\mathscr{P}^{F}(\succeq)\right)>0$ by taking $B=\Omega$ in Lemma 2 .

\footnotetext{
${ }^{12}$ See [17], [24] and [12].

${ }^{13}$ In fact, it is easy to find examples in which $\rho\left(\mathscr{P}^{W}(\succeq)\right)<\rho\left(\Omega \backslash \mathscr{P}^{W}(\succeq)\right)$, so betting on $\Omega \backslash \mathscr{P}^{W}(\succeq)$ is strictly preferred to betting on $\mathscr{P}^{W}(\succeq)$.
} 


\section{Generalizing Nash equilibrium}

There has been considerable recent interest in modelling the strategic behavior of CEU and MEU decision-makers. In particular, non-neutral attitudes to uncertainty seem intuitively likely to be important in strategic decision problems. ${ }^{14}$ Several generalizations of Nash equilibrium to the CEU and MEU domains have therefore been proposed. Typically, a subjective interpretation of equilibrium is adopted - that is, equilibrium beliefs, rather than equilibrium strategies, are identified. Various epistemic constructs are employed for this purpose, leading to several alternative notions of equilibrium. For example, Klibanoff [18] and Lo [19] use Savage belief, ${ }^{15}$ while Marinacci [21] and Mukerji and Shin [25] use weak belief.

Dow and Werlang [8], Mukerji [23] and Eichberger and Kelsey [9] consider normal form games played by CEU maximizers, and use supports of the associated capacities to construct player beliefs: "an event is known when it contains a support" ([8, p.319]). The possible existence of multiple supports causes the logic of this epistemic model to depart significantly from that of the other models mentioned in the present paper. This point is discussed at some length in section 5 of [8].

Lo, in his 1999 paper, has criticized these various generalizations of Nash equilibrium. On the one hand, he argues that those based on Savage belief are too conservative, while those based on weak belief or Dow and Werlang's epistemic model are too radical in their predictions. Lo proposes an alternative solution concept - cautious equilibrium - that is in the spirit of proper equilibrium [26], and steers an intermediate course between these two extremes. The notion of firm belief allows us to achieve a similar compromise, but without having to abandon the simple logic of Nash.

To define our new equilibrium, let us first introduce the following notation for finite, two-player normal form games:

$$
G=\left\langle S_{1}, S_{2}, g: S_{1} \times S_{2} \rightarrow X \times X\right\rangle .
$$

The (finite) set $S_{i}$ contains player $i$ 's strategies. The mapping $g$ assigns outcomes to the players. Thus, $g_{i}\left(s_{1}, s_{2}\right) \in X$ is player $i$ 's outcome if the strategy profile $\left(s_{1}, s_{2}\right)$ is played. Using $g$, it is obvious that we may identify each $s_{i} \in S_{i}$ with an act in $X^{S_{j}}(j \neq i)$.

We shall assume that player $i$ 's strategic behavior is determined by some preference ordering $\succeq_{i}$ over $X^{S_{j}}$. Player $i$ will therefore choose one of the strategies in the set

$$
B R_{i}\left(\succeq_{i}\right):=\left\{s_{i} \in S_{i} \mid s_{i} \succeq_{i} \hat{s}_{i} \quad \forall \hat{s}_{i} \in S_{i}\right\} .
$$

Informally, an equilibrium for $G$ is a pair of rational choice behaviors, such that each player's behavior is based on correct beliefs about her rival's strategic preferences. This informal description motivates the following definition:

\footnotetext{
14 For experimental evidence to this effect, see [4].

15 However, while Lo requires players to Savage believe that rivals always use best responses, Klibanoff only requires Savage belief in the possibility of rivals using best responses.
} 
Definition 4 A pair of preferences $\left(\succeq_{1}, \succeq_{2}\right)$ is a firm equilibrium for $G$ if, for each $i, j \in\{1,2\}$ with $j \neq i$ :

1. $\succeq_{i}$ is a biseparable ordering on $X^{S_{j}}$ compatible with a continuous $u$; and

2. $\mathscr{P}^{F}\left(\succeq_{i}\right) \subseteq B R_{j}\left(\succeq_{j}\right)$.

If each $\succeq_{i}$ is within the SEU class, then $\mathscr{P}^{F}\left(\succeq_{i}\right)=\mathscr{P}\left(\succeq_{i}\right)$, and we obtain an ordinary Nash equilibrium, though described through the preferences, rather than the subjective probabilities in their SEU representations. ${ }^{16}$ In this formulation of equilibrium, we do not exogenously fix the players' utility functions over constant acts. If these are fixed (and commonly known), this information would impose an additional exogenous restriction on equilibrium preferences.

Lo [20] uses the game in Figure 1 to assess the performance of several generalizations of Nash equilibrium against intuition.

2

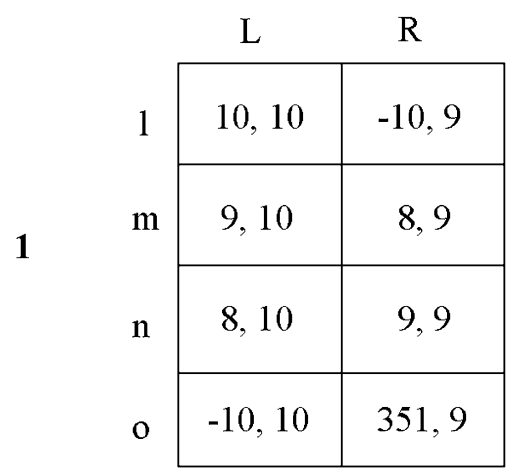

Figure 1

Lo argues that $m$ is a reasonable strategy for a "cautious" player 1 in this game, who "expects" player 2 to be rational, but is nevertheless perturbed by the Knightian uncertainty surrounding the formation of this expectation. ${ }^{17}$ However, $n$ is not a reasonable strategy for player 1 , since the expectation of rational behavior on the part of player 2 should intuitively make $m$ a better response than $n$. Therefore, Lo concludes that player 1 might use $l$ or $m$ in equilibrium, but not $n$.

Lo's own beliefs equilibrium [19], and Mukerji and Shin's ([25]) Nash equilibrium under Knightian uncertainty, are too conservative in this regard, as they only admit $l$ as an equilibrium strategy for player 1 . However, the solution concepts of Dow and Werlang ([8]), Mukerji [23], Klibanoff ([18, p.8]) and Marinacci ([21]) are too "weak", as they admit $n$ (as well as $l$ and $m$ ) as a possible equilibrium action. Eichberger and Kelsey's [9] equilibrium under uncertainty suffers the same "weakness", as it coincides with Dow and Werlang's solution concept for 2-player games.

\footnotetext{
${ }^{16}$ Cf. [20, p.628].

17 Dow and Werlang ([8, pp. 314-315]) make a similar point.
} 
Happily, firm equilibrium passes Lo's test. Since Nash equilibrium is a special case, $l$ may be supported as an equilibrium strategy for player 1. Moreover, so may strategy $m$. Let the payoffs in Figure 1 be cardinal utilities. Suppose that player 2 is an SEU maximizer, with a subjective probability supported on $\{m\}$; and player 1 is an MEU maximizer with

$$
C=\{p \in \Delta\{L, R\} \mid p(L) \in[0.9,1]\} .
$$

Using Corollary 3, it is straightforward to verify that the players' firm beliefs are generated by $\mathscr{P}^{F}\left(\succeq_{1}\right)=\{L\}$ and $\mathscr{P}^{F}\left(\succeq_{2}\right)=\{m\}$; while $B R_{1}\left(\succeq_{1}\right)=\{m\}$ and $B R_{1}\left(\succeq_{2}\right)=\{L\}$. Hence, $\left(\succeq_{1}, \succeq_{2}\right)$ constitutes a firm equilibrium for this game, and predicts the choice of $m$ by player $1 .^{18}$

Moreover, even if the payoffs in Figure 1 have only ordinal significance, it is impossible to support the strategy $n$ as a firm equilibrium prediction. Any firm equilibrium must satisfy $\mathscr{P}^{F}\left(\succeq_{1}\right)=\{L\}$. Suppose, then, that $\succeq_{1}$ are biseparable preferences with $\mathscr{P}^{F}\left(\succeq_{1}\right)=\{L\}$ and associated capacity $\rho_{1}$ in their canonical representation. By Theorem $2, \mathscr{P}^{F}\left(\succeq_{1}\right)=\{L\}$ requires $\rho_{1}(\{R\})=0$. If $\rho_{1}(\{L\})=$ 0 as well, then each singleton subset of $\{L, R\}$ is a support of $\rho_{1}$, so $\mathscr{P}^{F}\left(\succeq_{1}\right)=$ $\{L, R\}$. Hence, we must have $\rho_{1}(\{L\})>0$. But in this case, $m \succ_{1} n$, so $n$ can never be a best response for player 1 when $\mathscr{P}^{F}\left(\succeq_{1}\right)=\{L\}$.

We have already mentioned that Lo's cautious equilibrium is equally adept at handling the game in Figure 1, but employs a more complex game-theoretic logic. Moreover, there are games for which firm equilibrium and cautious equilibrium materially differ in their implications. For the case of CEU maximizers, Ryan [27] presents an example of a game with a firm equilibrium whose behavioral predictions are incompatible with any cautious equilibrium.

Finally, firm equilibrium is the first generalization of Nash equilibrium of which we are aware that is explicitly (and tractably) defined over the domain of biseparable preferences. This is significant, because admitting biseparable preferences strictly enlarges the range of equilibrium strategic behaviors relative to the CEU and MEU domains, as illustrated by the game in Figure 2 of [27].

\section{Concluding comments}

Firm belief is a useful epistemic model provided condition (6) is regarded as the appropriate behavioral test for the subjective possibility of states. This paper has presented both direct and indirect arguments to suggest that it is. For preferences in the CEU and MEU classes, for example, firm belief is less restrictive than Savage belief, but enjoys greater logical consistency than weak belief. Moreover, unlike weak belief, firm belief shares with Savage belief the desirable property that decision-makers always strictly prefer to "bet" on a believed event than its complement.

A major motivation for studying behavioral models of belief is their potential application to game theory. The previous section showed how firm belief might

${ }^{18}$ It also constitutes a cautious equilibrium - see [20, p. 629]. 
be used to extend the logic of Nash equilibrium to games whose players have biseparable preferences. This new solution concept - firm equilibrium - inherits the tractability of the representations of firm belief provided in Corollary 2, and treads an intermediate path between alternative generalizations of Nash.

Let us finally observe that the issue of equilibrium existence remains an interesting avenue for further research. Of course, equilibrium existence is only interesting if preferences are restricted so as to exclude some or all SEU orderings, since otherwise the existence of Nash equilibria settles the issue. However, one may sometimes wish to impose such restrictions - as in Marinacci's ([21, Definition 2]) notion of an ambiguous game. In such games, players are assumed to be CEU maximizers with convex capacities, and each event $A$ in a player $i$ 's state space is exogenously associated with an ambiguity level, $\Psi_{i}(A)$. If $v$ is player $i$ 's capacity, then it must satisfy $\Psi_{i}(A)=1-v(A)-v\left(A^{c}\right)$.

Marinacci ([21, p. 202]) asserts that weak belief is the only epistemic model compatible with the existence of equilibrium for arbitrary ambiguity levels. Indeed, this is a remarkable and intriguing property of weak belief. However, Marinacci's claim is certainly not fatal to alternative epistemic models, such as firm belief, since there are reasons to suppose that the exogenous imposition of arbitrary ambiguity levels is not always appropriate. First, it is difficult to make sense of ambiguity levels for non-convex capacities. Sometimes, as in Mukerji and Shin [25], it may be reasonable to restrict attention to such capacities, but this will not always be so.

Second, the basis for Marinacci's claim is the fact that weak belief is the only epistemic model for which ambiguity levels impose no restriction on what is believed. That is, for any event $A$ in player $i$ 's state space, and any exogenously imposed ambiguity levels $\Psi_{i}$, it is always possible to find preferences for $i$ that are compatible with $\Psi_{i}$ and that (weakly) believe $A .{ }^{19}$ This freedom of beliefs is clearly important to the "exogeneity" of the imposed restriction, as beliefs are the equilibrating variables in Marinacci's analysis, as they are in ours. However, while this commends the use of weak beliefs for the study of ambiguous games, its implications for alternative epistemic models is confined to the conclusion that ambiguity levels are not exogenous restrictions in such contexts. Indeed, the fact that ambiguity levels may be event-contingent intuitively suggests that such restrictions may be pushed too far.

Therefore, Marinacci's results, while far from fatal to the tractability of firm beliefs in the analysis of games, do suggest an important open question for further research: Do there exist preference classes which are (a) of independent interest; (b) described by conditions that can be convincingly established as exogenous; and (c) compatible with the existence of firm equilibria?

\footnotetext{
19 This fact may be verified using the technique employed in the proof of Marinacci's Theorem 1. It should be noted, however, that the result requires agnosticism regarding the beliefs of a decisionmaker with $\mathscr{P}^{W}(\succeq)=\emptyset$ : such preferences are implicitly taken to be compatible with any belief. This does not imply that Marinacci adopts the logically untenable position that such players believe anything; but simply that one cannot rule out, on the basis of such preferences, belief in any particular proposition.
} 


\section{Proof of Theorem 4.1}

First define $\geq_{L}$ to be the "not infinitely less likely than" relation. That is, $A \geq_{L} B$ if and only if it is not the case that $B \gg_{L} A$.

Definition 5 Given a preference ordering $\succeq$, the associated relation $\geq_{L}$ on $2^{\Omega}$ is transitive on nested sequences if $A \geq_{L} C$ whenever $A \subseteq B \subseteq C, A \geq_{L} B$ and $B \geq{ }_{L} C$.

Lemma 3 Let $\succeq$ be a complete, transitive and non-trivial ordering. If the associated relation $\geq_{L}$ on $2^{\Omega}$ is transitive on nested sequences, then it follows that $\mathscr{P}^{F}(\succeq) \neq \emptyset$.

Proof. Let

$$
\emptyset=E_{0} \subseteq E_{1} \subseteq \cdots \subseteq E_{n}=\Omega
$$

be such that $E_{k}$ contains exactly one more state than $E_{k-1}$ for each $k \geq 1$ (and hence $n=|\Omega|$ ). Notice that $\Omega \gg_{L} \emptyset$ by definition. Since $\geq_{L}$ is transitive on nested sequences, there must exist some $k \geq 1$ such that $E_{k} \gg_{L} E_{k-1}$. Therefore, $\mathscr{P}^{F}(\succeq)$ contains that state $\omega \in E_{k}$ which is excluded from $E_{k-1}$.

It therefore suffices to show that $\succeq$ is transitive on nested sequences when $\succeq$ possesses a lexicographic canonical representation.

Suppose $A \subseteq B \subseteq C, A \geq_{L} B$ and $B \geq_{L} C$. Then (7) implies

$$
[u(y)-u(z)] \rho(A) \geq^{n}[u(x)-u(z)] \rho(B)
$$

for some $x, y, z \in X$ with $x \succ z$; and

$$
[u(\hat{y})-u(\hat{z})] \rho(B) \geq^{n}[u(\hat{x})-u(\hat{z})] \rho(C)
$$

for some $\hat{x}, \hat{y}, \hat{z} \in X$ with $\hat{x} \succ \hat{z}$.

If $\rho(B)=\mathbf{0}$, then $\rho(A)=\mathbf{0}$ by the monotonicity of $\rho$, and $\rho(C)=\mathbf{0}$ by (16). Hence,

$$
[u(y)-u(z)] \rho(A)=[u(x)-u(z)] \rho(C)=\mathbf{0},
$$

which implies $A \geq_{L} C$.

Suppose that $\rho(B)>\mathbf{0}$. Letting

$$
\begin{aligned}
& k_{1}=u(y)-u(z), \\
& k_{2}=u(x)-u(z), \\
& k_{3}=u(\hat{y})-u(\hat{z}),
\end{aligned}
$$

and

$$
k_{4}=u(\hat{x})-u(\hat{z})
$$


monotonicity of $\rho$ and (15)-(16) imply $k_{1} \geq k_{2}>0$ and $k_{3} \geq k_{4}>0$.

If $k_{2} \geq k_{3}$, then

$$
[u(y)-u(z)] \rho(A) \geq^{n}[u(\bar{x})-u(z)] \rho(C)
$$

where $\bar{x}$ is chosen so that $u(\bar{x})-u(z)=k_{4}>0$. It is always possible to find such an $\bar{x}$, since $u(X)$ is an interval, ${ }^{20}$ and $u(y)-u(z)=k_{1} \geq k_{4}$. From (17) it follows that $A \geq_{L} C$.

Finally, consider the case $k_{3}>k_{2}>0$. From (15) and (16) we have

$$
k_{1} \rho(A) \geq^{n} k_{2} \rho(B) \geq^{n} \alpha k_{3} \rho(B) \geq^{n} \alpha k_{4} \rho(C),
$$

where $\alpha=k_{2} / k_{3} \in(0,1)$. Since $A \subseteq C$ and $\rho(C) \geq \rho(B)>\mathbf{0}$, it follows that $k_{1} \geq \alpha k_{4}>0$. Therefore, choose $\tilde{x}$ such that $u(\tilde{x})-u(z)=\alpha k_{4}>0$ and conclude

$$
[u(y)-u(z)] \rho(A) \geq^{n}[u(\tilde{x})-u(z)] \rho(C) .
$$

Hence $A \geq_{L} C$.

This completes the proof of Theorem 1 .

\section{References}

1. Anscombe, F. J., Aumann, R. J.: A definition of subjective probability. Annals of Mathematical Statistics 34, 199-205 (1963)

2. Blume, L., Brandenburger, A., Dekel, E.: Lexicographic probabilities and choice under uncertainty. Econometrica 59, 61-79 (1991a)

3. Blume, L., Brandenburger, A., Dekel, E.: Lexicographic probabilities and equilibrium refinements. Econometrica 59, 81-98 (1991b)

4. Camerer, C. F., Karjalainen, R.: Ambiguity-aversion and non-additive beliefs in non-cooperative games: experimental evidence. In: Munier, B., Machina, M. J. (eds.) Models and experiments in risk and rationality. Dordrecht: Kluwer Academic 1994

5. Casadesus-Masanell, R., Klibanoff, P., Ozdenoren, E.: Maxmin expected utility over Savage acts with a set of priors. Journal of Economic Theory 92, 35-65 (2000)

6. Choquet, G.: Theory of capacities. Annales de L'Institut Fourier V, 131-295 (1953-54)

7. Dempster, A. P.: Upper and lower probabilities induced by a multivalued mapping. Annals of Mathematical Statistics 38, 325-339 (1967)

8. Dow, J., Werlang, S. R., Da C.: Nash equilibrium under Knightian uncertainty: breaking down backward induction. Journal of Economic Theory 64, 305-324 (1994)

9. Eichberger, J., Kelsey, D.: Non-additive beliefs and strategic equilibria. Games and Economic Behavior 30, 183-215 (2000)

10. Ellsberg, D.: Risk, ambiguity and the Savage axioms. Quarterly Journal of Economics 75, 643669 (1961)

11. Epstein, L. G.: A definition of uncertainty aversion. Review of Economic Studies 66, 579-608 (1999)

12. Ghirardato, P.: Coping with ignorance: unforeseen contingencies and non-additive uncertainty. Economic Theory 17, 247-276 (2001)

13. Ghirardato, P., Marinacci, M.: Ambiguity made precise: a comparative foundation. Social Science WP 1026, California Institute of Technology (2000a)

14. Ghirardato, P., Marinacci, M.: Risk, ambiguity and the separation of utility and beliefs. Social Science WP 1085, California Institute of Technology (2000b)

${ }^{20}$ Recall that $X$ is connected and $u$ continuous. 
15. Gilboa, I.: Expected utility with purely subjective non-additive probabilities. Journal of Mathematical Economics 16, 65-88 (1987)

16. Gilboa, I., Schmeidler, D.: Maxmin expected utility with a non-unique prior. Journal of Mathematical Economics 18, 141-153 (1989)

17. Gilboa, I., Schmeidler, D.: Additive representations of non-additive measures and the Choquet Integral. Annals of Operations Research 52, 43-65 (1994)

18. Klibanoff, P.: Uncertainty, decision and normal-form games. Unpublished manuscript, Kellogg Graduate School of Management, Northwestern University (1996)

19. Lo, K. C.: Equilibrium in beliefs under uncertainty. Journal of Economic Theory 71, 443-484 (1996)

20. Lo, K.C.: Nash equilibrium without mutual knowledge of rationality. Economic Theory 14, 621-633 (1999)

21. Marinacci, M.: Ambiguous games. Games and Economic Behavior 31, 191-219 (2000)

22. Morris, S.: Alternative definitions of knowledge. In: Bacharach, M. O. L. et al. (eds.) Epistemic logic and the theory of games and decisions. Dordrecht: Kluwer Academic 1997

23. Mukerji, S.: A theory of play for games in strategic form when rationality is not common knowledge. Unpublished manuscript, University of Southampton (1995)

24. Mukerji, S.: Understanding the nonadditive probability decision model. Economic Theory 9 , 23-46 (1997)

25. Mukerji, S., Shin, H. S.: Equilibrium departures from common knowledge in games with nonadditive expected utility. Unpublished manuscript, University of Southampton (1998)

26. Myerson, R.: Refinements of the Nash equilibrium concept. International Journal of Game Theory 1, 73-80 (1978)

27. Ryan, M. J.: Some properties of firm equilibrium: A note. Unpublished manuscript, The Australian National University (2000). Available at http://ecocomm.anu.edu.au/economics/staff/ryan/ ryan.html

28. Sarin, R., Wakker, P.: A simple axiomatization of nonadditive expected utility. Econometrica 60(6), 1255-1272 (1992)

29. Savage, L. J.: The foundations of statistics. New York: Wiley 1954

30. Schmeidler, D.: Subjective probability and expected utility without additivity. Econometrica 57(3), 571-587 (1989)

31. Wakker, P. P.: Testing and characterizing properties of nonadditive measures through violations of the sure-thing principle. Econometrica (forthcoming)

32. Yaari, M. E.: Some remarks on measures of risk aversion and on their uses. Journal of Economic Theory 1, 315-329 (1969) 